\title{
Research Article \\ Derivation of Conditions for the Normal Gain Behavior of Conical Horns
}

\author{
Chin Yeng Tan, Krishnasamy T. Selvan, and Yew Meng Koh
}

Received 12 January 2007; Revised 23 May 2007; Accepted 3 November 2007

Recommended by Deb Chatterjee

\begin{abstract}
Monotonically increasing gain-versus-frequency pattern is in general expected to be a characteristic of aperture antennas that include the smooth-wall conical horn. While optimum gain conical horns do naturally exhibit this behavior, nonoptimum horns need to meet certain criterion: a minimum axial length for given aperture diameter, or, alternatively, a maximum aperture diameter for the given axial length. In this paper, approximate expressions are derived to determine these parameters.
\end{abstract}

Copyright (c) 2007 Chin Yeng Tan et al. This is an open access article distributed under the Creative Commons Attribution License, which permits unrestricted use, distribution, and reproduction in any medium, provided the original work is properly cited.

\section{INTRODUCTION}

A monotonically increasing gain with the operating frequency, referred to as the "normal" gain behavior in this paper, is a salient feature of aperture antennas [1], that include the conical and the pyramidal horns. While optimum horns satisfy this criterion, nonoptimum horns do not automatically exhibit the normal gain behavior. In [2], Selvan has derived an approximate expression for the minimum axial length required by pyramidal horns of given aperture to display the normal gain behavior. Following [2], in this paper, expressions are derived for estimating the minimum axial length for a given optimum aperture diameter, and maximum aperture diameter for the given optimum axial length for the case of the conical horn in order that it displays the normal gain behavior.

This paper proceeds as follows. In Section 2, the approximate conical horn gain formula [3] used in the derivations is presented. The derivation of minimum axial length for the given optimum aperture diameter is discussed in Section 3, while Section 4 presents the derivation of an expression for determining the maximum aperture diameter for the given optimum axial length. Section 5 concludes the paper.

\section{CONICAL HORN GAIN FORMULA}

With reference to the geometry of the conical horn shown in Figure 1, the gain of the conical horn is given by [4]

$$
G(\mathrm{~dB})=20 \log \left(\frac{D \pi}{\lambda}\right)-\mathrm{LF}
$$

where $\lambda$ is the free-space wavelength. The loss factor LF that accounts for the reduction in gain due to aperture efficiency is given by

$$
\mathrm{LF} \approx 0.8-1.71 s+26.25 s^{2}-17.79 s^{3}
$$

with

$$
s=\frac{D^{2}}{8 \lambda l}
$$

as the aperture phase error. An improved approximation for LF is [3]

$$
\mathrm{LF} \approx 0.75+0.66 s+9.4 s^{2}+6.8 s^{3} .
$$

In what follows, we make use of this improved loss factor expression.

Since we are interested in the frequency-versus-gain behavior of the horn, (1) can be more conveniently rewritten as

$$
g(f)=\left(\frac{D \pi}{c}\right)^{2} f^{2} 10^{-(\mathrm{LF} / 10)},
$$

where $c$ is the speed of light.

\section{MINIMUM AXIAL LENGTH}

To derive the minimum axial length $L_{\min }$ such that $g(f)$ remains a monotonically increasing function in the desired frequency range $f_{1} \leq f \leq f_{2},(4)$ and (5) have to be rewritten as a function of $f$. Thus, (3) can be written as

$$
s=y\left(\frac{f}{l}\right),
$$




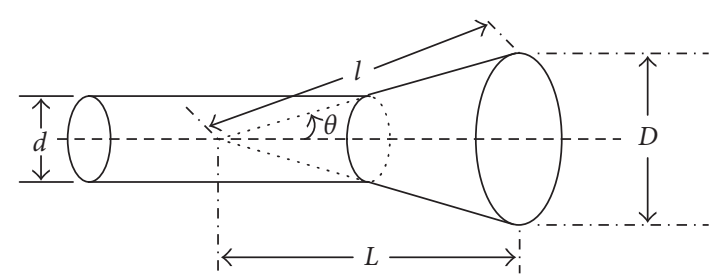

Figure 1: Geometry of smooth-walled conical horn.

where

$$
y=\frac{D^{2}}{8 c} .
$$

By substituting (6) and (7) into (4), the loss factor can be expressed as a function of frequency $f$ :

$$
\mathrm{LF}=6.8 y^{3}\left(\frac{f^{3}}{l^{3}}\right)+9.4 y^{2}\left(\frac{f^{2}}{l^{2}}\right)+0.66 y\left(\frac{f}{l}\right)+0.75 .
$$

Using (8), the gain formula in (5) can be expressed as a function of frequency, $f$, as

$$
g(f)=\left(\frac{D \pi}{c}\right)^{2} f^{2}\left\{10^{-\left[\alpha_{3}\left(f^{3} / l^{3}\right)+\alpha_{2}\left(f^{2} / l^{2}\right)+\alpha_{1}(f / l)+0.075\right]}\right\}
$$

where

$$
\alpha_{3}=0.68 y^{3}, \quad \alpha_{2}=0.94 y^{2}, \quad \alpha_{1}=0.066 y .
$$

In order to be a monotonically increasing function in the range of $f_{1} \leq f \leq f_{2}$, the derivative of $g(f)$ must be greater than zero in this range [2]. Mathematically,

$$
\ln 10\left[-3 \alpha_{3}\left(\frac{f^{3}}{l^{3}}\right)-2 \alpha_{2}\left(\frac{f^{2}}{l^{2}}\right)-\alpha_{1}\left(\frac{f}{l}\right)\right]+2>0 .
$$

By substituting $f=z l$ and dividing by $-3 \alpha_{3}$, (11) becomes

$$
Z^{3}+\left(\frac{2 \alpha_{2}}{3 \alpha_{3}}\right) Z^{2}+\left(\frac{\alpha_{1}}{3 \alpha_{3}}\right) Z-\frac{2}{3 \alpha_{3} \ln 10}<0 .
$$

Equation (12) needs to be satisfied so that $g(f)$ monotonically increases in the operating frequency band, $f_{1} \leq f \leq$ $f_{2}$. Equation (12) has three real roots with only one positive root for "real" horns [2]. This positive root of (11) is given by $[2,5]$

$$
Z_{1}=\left(s_{1}+s_{2}\right)-\frac{2 \alpha_{2}}{9 \alpha_{3}}
$$

where

$$
\begin{aligned}
s_{1} & =\left[p+\sqrt{q^{3}+p^{2}}\right]^{1 / 3}, \\
s_{2} & =\left[p-\sqrt{q^{3}+p^{2}}\right]^{1 / 3}, \\
p & =\frac{1}{6}\left[\frac{2 \alpha_{1} \alpha_{2}}{9 \alpha_{3}^{2}}+\frac{2}{\alpha_{3} \ln 10}\right]-\frac{1}{27}\left(\frac{2 \alpha_{2}}{3 \alpha_{3}}\right)^{3}, \\
q & =\frac{1}{9}\left[\frac{\alpha_{1}}{\alpha_{3}}-\left(\frac{2 \alpha_{2}}{3 \alpha_{3}}\right)^{2}\right] .
\end{aligned}
$$

Taking $f_{2}$ to be the upper frequency, we have the minimum slant length, $l_{\min }$, as

$$
l_{\min }=\frac{f_{2}}{Z_{1}} .
$$
is

Therefore, the corresponding minimum axial length $L_{\min }$

$$
L_{\min }=\sqrt{l_{\min }^{2}-\frac{D^{2}}{4}} .
$$

The minimum axial length calculated from (16) will always be less than the optimum axial length [2]. For illustration, we consider a commercially available optimum-gain $K u$-band conical horn with the dimensions $d=1.6764 \mathrm{~cm}$, $D=10.16 \mathrm{~cm}$, and $L=17.78 \mathrm{~cm}$. For this horn, the minimum axial length for normal gain behavior predicted by (16) is $13.68 \mathrm{~cm}$. Figure 2 (a) presents the gain as a function of frequency for this horn, as predicted by the improved approximate gain formula [3] and the CST Microwave Studio simulation software. The two predictions are seen to show good agreement with each other. In this case, the gain behavior is seen to be "normal." When the axial length, however, is reduced to $12.68 \mathrm{~cm}$ (which is less than the minimum axial length), the gain starts dropping at around $16.8 \mathrm{GHz}$, as demonstrated in Figure 2(b).

\section{MAXIMUM APERTURE DIAMETER}

The maximum aperture diameter $D_{\max }$ can be derived in a way similar to the one used for minimum axial length, Section 3. To begin with, the phase errors as given in (3) can be expressed as

$$
s=y x f
$$

where

$$
y=\frac{1}{4 c}, \quad x=D \sin \left[\tan ^{-1}\left(\frac{D}{2 L}\right)\right] .
$$

By substituting (17), and (18) into (4), the loss factor can be expressed in terms of frequency $f$ as

$$
\mathrm{LF}=6.8 y^{3}(x f)^{3}+9.4 y^{2}(x f)^{2}+0.66 y(x f)+0.75 .
$$

Upon substituting (19) into (5), the conical horn gain formula can be rewritten as

$$
g(f)=\left(\frac{D \pi}{c}\right)^{2} f^{2}\left\{10^{-\left[\alpha_{3}(x f)^{3}+\alpha_{2}(x f)^{2}+\alpha_{1}(x f)+0.075\right]}\right\},
$$

where

$$
\alpha_{3}=0.68 y^{3}, \quad \alpha_{2}=0.94 y^{2}, \quad \alpha_{1}=0.066 y .
$$

With the similar condition as explained for (11), the derivative of $g(f)$ can be written as

$$
\ln 10\left[-3 \alpha_{3}(x f)^{3}-2 \alpha_{2}(x f)^{2}-\alpha_{1}(x f)\right]+2>0 .
$$




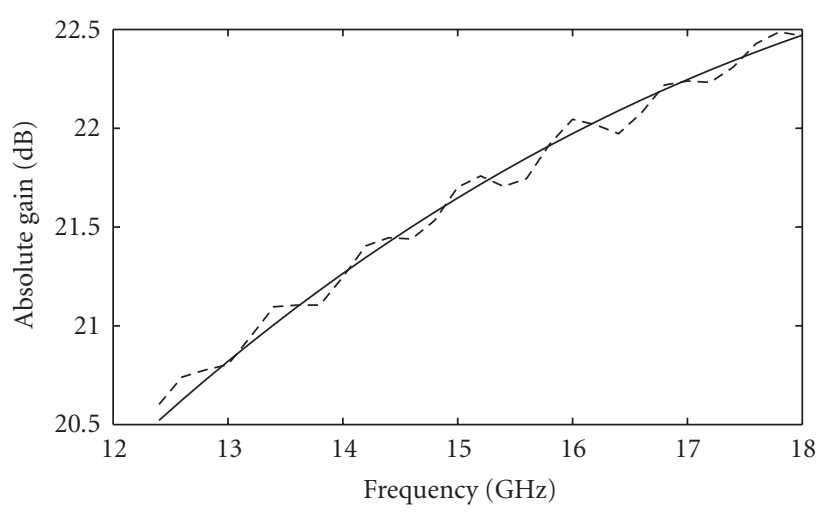

(a)

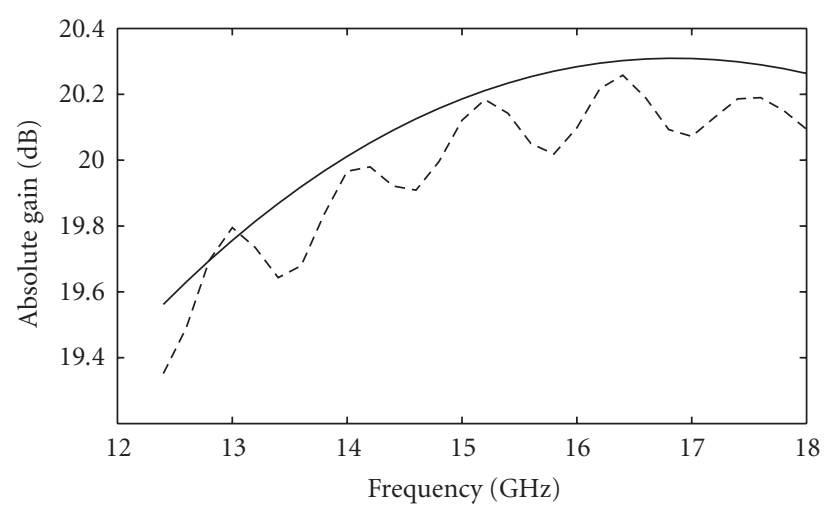

(b)

FIGURE 2: Gain pattern for a $K u$-band conical horn. (a) $L=17.78 \mathrm{~cm}$ (optimum axial length), (b) $L=12.68 \mathrm{~cm}$ (less than minimum axial length required for normal gain pattern). The solid line is obtained by using approximate gain formula [3]. The dashed line is simulated by using CST Microwave Studio.

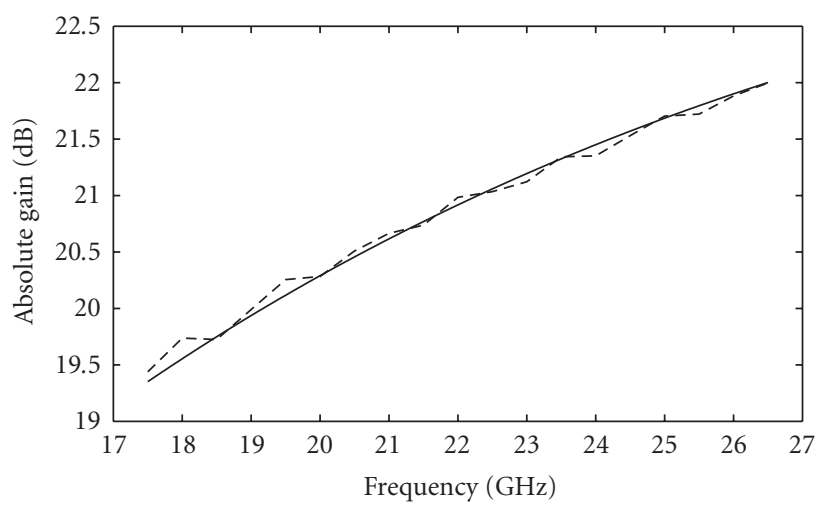

(a)

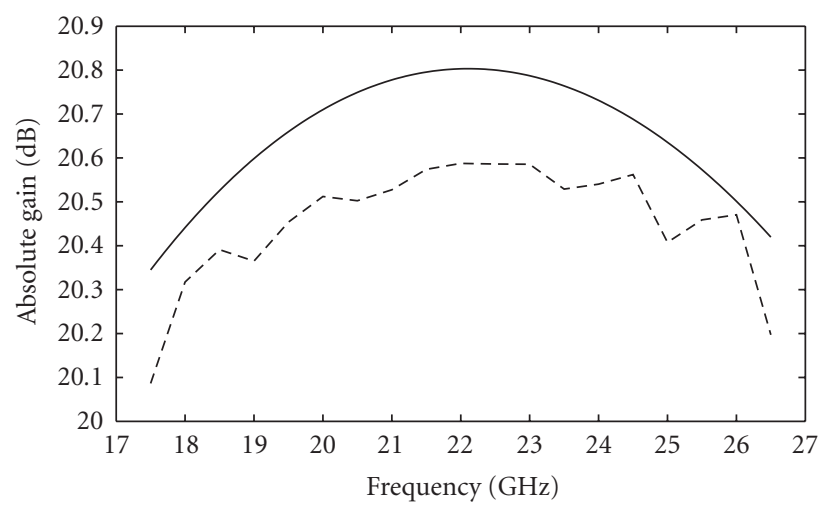

(b)

Figure 3: Gain pattern for a $K$-band conical horn. (a) $D=6.02 \mathrm{~cm}$ (optimum aperture diameter), (b) $D=8.20 \mathrm{~cm}$ (greater than maximum aperture diameter required for normal gain pattern). The solid line is obtained by using approximate gain formula [3]. The dashed line is simulated by using CST Microwave Studio.

By substituting $f=z / x$ and dividing by $-3 \alpha_{3}$, (22) becomes

$$
Z^{3}+\left(\frac{2 \alpha_{2}}{3 \alpha_{3}}\right) Z^{2}+\left(\frac{\alpha_{1}}{3 \alpha_{3}}\right) Z-\frac{2}{3 \alpha_{3} \ln 10}<0 .
$$

Equation (22) has three real roots, with just one positive real root for physical horns. This positive real root is given by $[2,5]$

$$
Z_{1}=\left(s_{1}+s_{2}\right)-\frac{2 \alpha_{2}}{9 \alpha_{3}}
$$

where

$$
\begin{aligned}
s_{1} & =\left[p+\sqrt{q^{3}+p^{2}}\right]^{1 / 3}, \\
s_{2} & =\left[p-\sqrt{q^{3}+p^{2}}\right]^{1 / 3}, \\
p & =\frac{1}{6}\left[\frac{2 \alpha_{1} \alpha_{2}}{9 \alpha_{3}^{2}}+\frac{2}{\alpha_{3} \ln 10}\right]-\frac{1}{27}\left(\frac{2 \alpha_{2}}{3 \alpha_{3}}\right)^{3}, \\
q & =\frac{1}{9}\left[\frac{\alpha_{1}}{\alpha_{3}}-\left(\frac{2 \alpha_{2}}{3 \alpha_{3}}\right)^{2}\right] .
\end{aligned}
$$

If $f_{1}$ is the lower frequency and $f_{2}$ is the upper frequency, then the maximum aperture diameter, $D_{\max }$, is given as

$$
D_{\max } \sin \left[\tan ^{-1}\left(\frac{D_{\max }}{2 L}\right)\right]=\frac{Z_{1}}{f_{2}} .
$$

The maximum aperture diameter calculated from (26) will always be greater than the optimum aperture diameter. For illustration, we consider a commercially available optimum-gain $K$-band conical horn with the dimensions $d$ $=1.15 \mathrm{~cm}, D=6.02 \mathrm{~cm}$, and $L=10.92 \mathrm{~cm}$. For this horn, the maximum aperture diameter for normal gain behavior predicted by (26) is $7.45 \mathrm{~cm}$. Figure 3(a) presents the gain as a function of frequency for this horn, as predicted by the improved approximate gain formula [3] and the CST Microwave Studio simulation software. Again, good agreement is noted between the predictions made by simulation and theory. In this case, the gain behavior is seen to be "normal." When the aperture diameter, however, is increased to $8.20 \mathrm{~cm}$ (which is greater than the maximum aperture diameter), the 
gain starts dropping at around $22.1 \mathrm{GHz}$, as demonstrated in Figure 3(b).

\section{CONCLUSION}

Approximate expressions were derived for estimating the minimum axial length for the given optimum diameter and maximum aperture diameter for the given optimum axial length for nonoptimum conical horns to exhibit normal gain behavior. These expressions might be useful when short horns offering monotonically increasing gain-versusfrequency pattern are desired to be designed.

\section{REFERENCES}

[1] W. L. Stutzman and G. A. Thiele, Antenna Theory and Design, John Wiley \& Sons, New York, NY, USA, 2nd edition, 1998.

[2] K. T. Selvan, "Derivation of a condition for the normal gain behavior of pyramidal horns," IEEE Transactions on Antennas and Propagation, vol. 48, no. 11, pp. 1782-1784, 2000.

[3] C. Y. Tan and K. T. Selvan, "An improved approximate gain formula for conical horn," Microwave and Optical Technology Letters, vol. 49, no. 4, pp. 971-973, 2007.

[4] C. A. Balanis, Antenna Theory: Analysis and Design, John Wiley \& Sons, Hoboken, NJ, USA, 3rd edition, 2005.

[5] M. Abramowitz and I. A. Stegun, Eds., Handbook of Mathematical Functions with Formulas, Graphs and Mathematical Tables, Dover, New York, NY, USA, 1977.

\section{AUTHOR CONTACT INFORMATION}

Chin Yeng Tan: Faculty of Engineering and Computer Science, The University of Nottingham Malaysia Campus, Jalan Broga, Semenyih 43500, Selangor Darul Ehsan, Malaysia; chinyeng.tan@nottingham.edu.my

Krishnasamy T. Selvan: Faculty of Engineering and Computer Science, The University of Nottingham Malaysia Campus, Jalan Broga, Semenyih 43500, Selangor Darul Ehsan, Malaysia; krishnasamy.selvan@nottingham.edu.my

Yew Meng Koh: Faculty of Engineering and Computer Science, The University of Nottingham Malaysia Campus, Jalan Broga, Semenyih 43500, Selangor Darul Ehsan, Malaysia; koh.yew-meng@nottingham.edu.my 

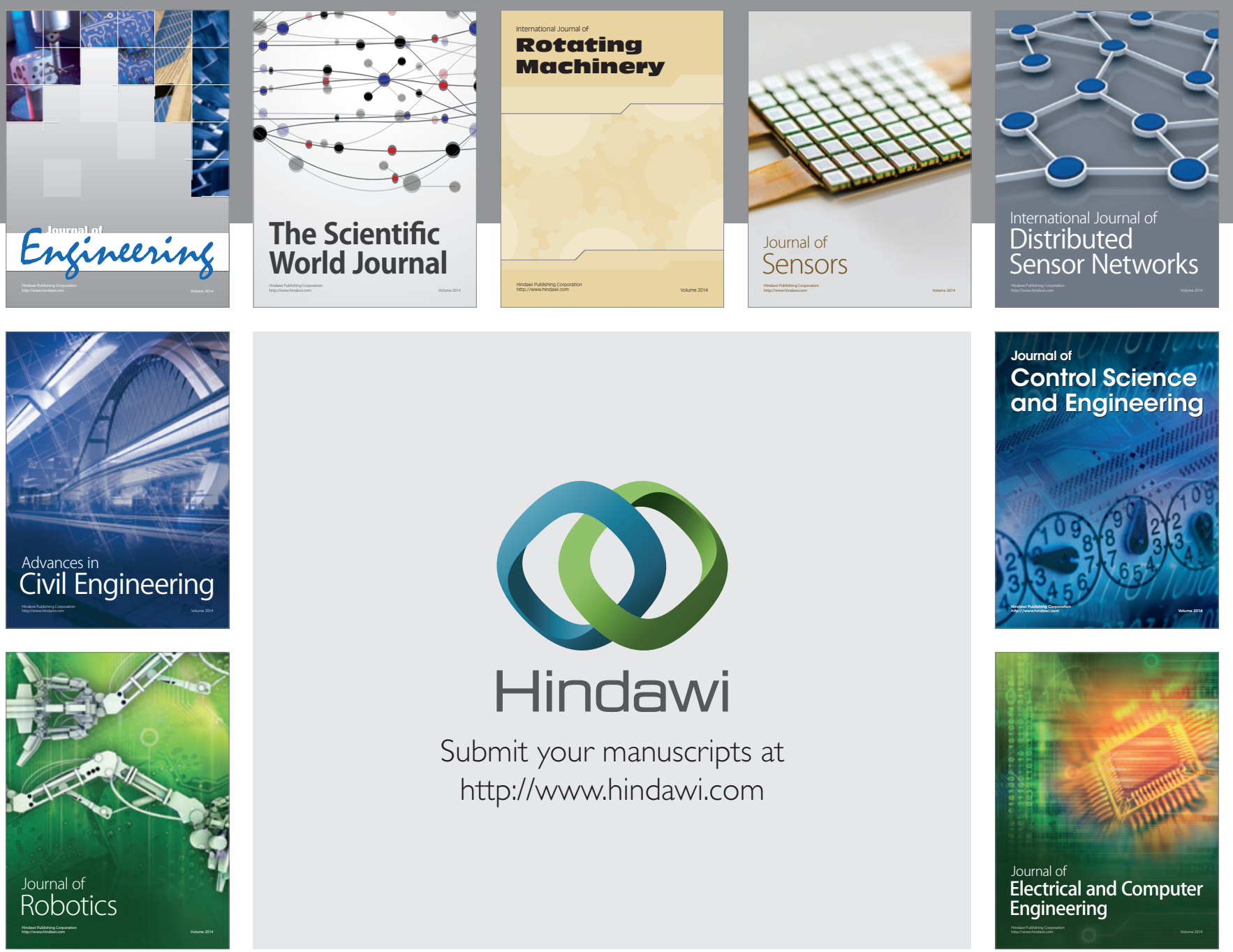

Submit your manuscripts at

http://www.hindawi.com
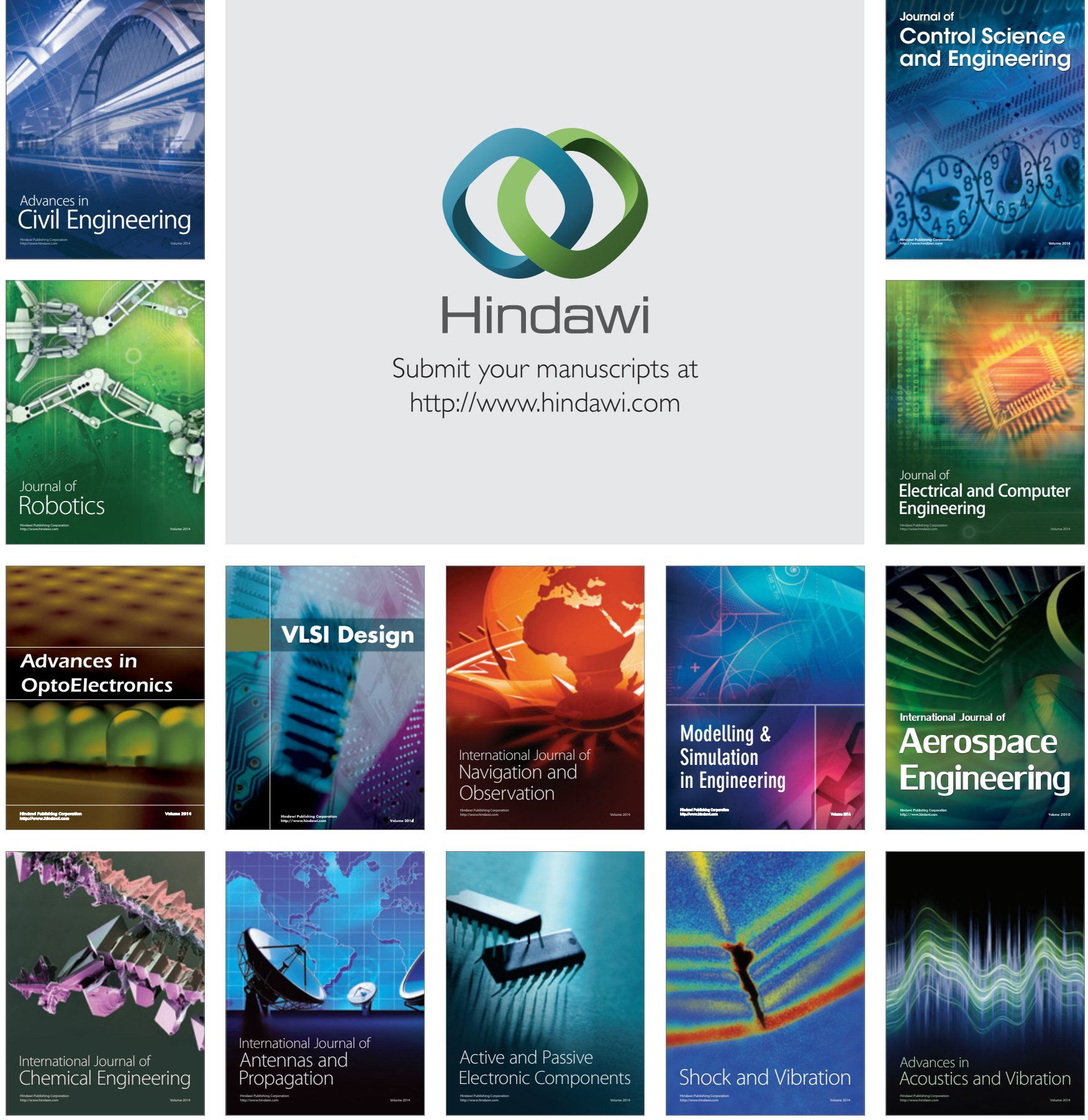